\title{
Coaxial Probe Fed Modified Sierpinski Fractal Antenna for Wireless Applications
}

\author{
Yadwinder Kumar
}

\begin{abstract}
A modified Sierpinski fractal antenna has been designed for wireless applications. The designed antenna exhibits multiple resonance behavior due to the basic attributes of the fractal shapes. The proposed antenna has planar, compact in size and is suitable for various wireless applications. It is designed on the Flame Retardant epoxy board substrate (FR4), which is very easily available, light in weight and has less cost. IFS (Iterated Function System) methodology is accustomed to generate the complex fractal layout using the scripting methodology (.vbs) in the HFSS simulator. Scripting method provides a straight forward solution to generate complicated fractal structures by generating code in MATLAB. The proposed antenna resonates at five different frequencies $1.859 \mathrm{GHz}, 3.623 \mathrm{GHz}, 5.929 \mathrm{GHz}$, $9.095 \mathrm{GHz}$ and $9.547 \mathrm{GHz}$ with smart values of return loss up to $26 \mathrm{~dB}$. It additionally demonstrates good radiation properties and has VSWR values less than two for all resonating frequencies. Radiation characteristics are displayed by $2 D$ and $3 D$ radiation patterns. It also has an low profile value of Gain of $3 \mathrm{~dB}$.
\end{abstract}

Keywords : Sierpinski Gasket, Fractal Antenna, Multiband, IFS, Return Loss, FR4, VSWR.

\section{INTRODUCTION}

There has been widespread use of wireless communication through recent years. This has opened new doors for researchers to explore bigger prospects of exploit multi-band antennas using fractal shapes. Planar microstrip antennas are the foremost vital choice by researchers to use multiple resonance properties of fractal shapes [1] as radiation structures. Self Similarity, space-filling and miniaturization are some vital properties transmissible by fractal shapes that may be accustomed to obtain compact and multiband antennas [2]. Many fractal geometries like Koch curve, Cantor set, Hilbert curve, Sierpinski gasket and Minkowski fractal [3] are usually utilized in designing fractal antennas that are accustomed acquire antennas that are compact in size and has multiband characteristics [4][5]. These are helpful in developing new and innovative antenna structures - Multiple resonant frequencies among a single compact device have given researchers a daring task to design and develop a multiband antenna capable of exhibiting multiband behavior [6]. Fractal geometries and shapes can offer an exposition to the present drawback. Benoit Mandelbrot [7] proposed fractal geometries in 1951, that were later used extensively to notice and design numerous antenna structures. Fractal structures boast distinctive options like self-similarity that facilitate to attain compactness and multiband/wideband behavior [8].

\footnotetext{
Revised Manuscript Received on December 30, 2019.

* Correspondence Author

Yadwinder Kumar, ECE Department, YCOE (Punjabi University), Talwandi Sabo, India.
}

(C) The Authors. Published by Blue Eyes Intelligence Engineering and Sciences Publication (BEIESP). This is an open access article under the CC BY-NC-ND license (http://creativecommons.org/licenses/by-nc-nd/4.0/)
Self-similarity can be consummate by applying an outsized range of iterations utilizing multiple cuts of reference shapes. The Koch curve and the Sierpinski curve [10] are some usually used geometry in fractal antenna design. Using these popular fractal shapes, varied items of researches are developed by varied ways to get multiple resonances, combining 2 or a lot of fractal geometries and modification of existing geometry are a number of the ways in which to attain this. [4] Fractals provides massive surface areas, separation, edges, segments, etc. during a restricted area. They are formed by periodic processes which results in lot of compactness and multiple resonances is consummated by availing broken framework as collated to other patch layouts [10].

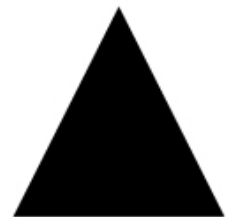

(a)

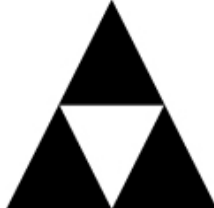

(b)

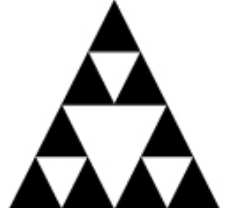

(c)
Figure 1. Sierpinski Gasket fractal with various iterations.

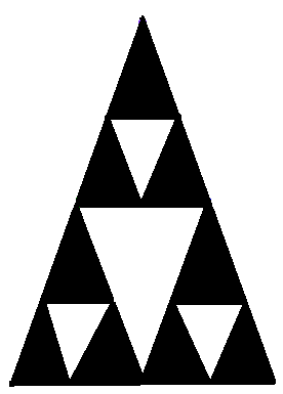

Figure 2. Proposed modified Sierpinski gasket fractal Sierpinski fractal is a widely used fractal structure in planar as well as non-planar antennas configurations[5][11].

\section{ANTENNA LAYOUT}

In this analysis, the suggested fractal antenna is intended by modifying the standard Sierpinski gasket with all equal sides and a widening angle of $60^{\circ}$. As shown in Figure 1. Figure 2 shows the modified Sierpinski with a flare angle of $50^{\circ}$ and therefore the different two angles at $65^{\circ}$ each. It has only two equal sides leading to isosceles triangle design. The Sierpinski gasket is one of the wide used fractal shapes used as antenna in several wireless applications [12]. Some vital factors needed to design the Sierpinski gasket fractal are the iteration range, scaling factor, triangle height and widening angle consisting of concatenation of scaled triangles with a scale factor of $\zeta=0.5$ [13]. 


\section{Coaxial Probe Fed Modified Sierpinski Fractal Antenna for Wireless Applications}

The proposed modified Sierpinski fractal structure has been obtained from the Iterated function system (IFS) [14], a very worthwhile calculation instrument used to narrate scaling, rotation, and translation of fractal layouts [15].

Various such operations can be performed by the following function:

$$
W\left(\begin{array}{l}
x \\
y
\end{array}\right)=\left(\begin{array}{ll}
a & b \\
c & d
\end{array}\right)\left(\begin{array}{l}
x \\
y
\end{array}\right)+\left(\begin{array}{l}
e \\
f
\end{array}\right)
$$

Here $a, b, c$ and $d$ control the rotation and scaling while $e$ and $f$ control linear shift [14].

$$
\begin{aligned}
& W_{1}\left(\begin{array}{l}
x \\
y
\end{array}\right)=\left(\begin{array}{cc}
-1.0 / 2.0 & 0 \\
0 & 1.0 / 2.0
\end{array}\right)\left(\begin{array}{l}
x \\
y
\end{array}\right)+\left(\begin{array}{c}
1 / 2 \\
0
\end{array}\right) \\
& W_{2}\left(\begin{array}{l}
x \\
y
\end{array}\right)=\left(\begin{array}{cc}
1.0 / 4.0 & 7 / 20 \\
67 / 125 & -1.0 / 4.0
\end{array}\right)\left(\begin{array}{l}
x \\
y
\end{array}\right)+\left(\begin{array}{c}
1 / 2 \\
0
\end{array}\right) \\
& W_{3}\left(\begin{array}{l}
x \\
y
\end{array}\right)=\left(\begin{array}{cc}
1 / 4 & -7 / 20 \\
-67 / 125 & -1.0 / 4.0
\end{array}\right)\left(\begin{array}{l}
x \\
y
\end{array}\right)+\left(\begin{array}{c}
1 / 2 \\
1.072
\end{array}\right)
\end{aligned}
$$

If $A$ be the starting structure. Various transformations on $A$, can be revealed as follows:

$$
W(A)=\bigcup_{n=1}^{N} W n(A)
$$

The Sierpinski fractal geometry can be obtained from the Hutchinson operator ' $W$ ' . Figure 3 is the final structure obtained by using scripting method in the HFSS electromagnetic simulator. The proposed antenna layout is sketched on the FR4 substrate having values of $\mathscr{E}_{\mathrm{r}}=4.4$, tan $\delta=0.02, h=1.6 \mathrm{~mm}$ and area of $54 \times 46 \mathrm{~mm}^{2}$. Values of $\mathcal{E}_{\text {reff }}$ and impedance $Z_{0}$ can be calculated using equations (6) and (7) [4].

$$
\begin{aligned}
\varepsilon_{\text {reff }} & =\frac{\varepsilon_{\mathrm{r}}+1}{2}+\frac{\varepsilon_{\mathrm{r}}-1}{2}[1 / \sqrt{1+12 h / w}] \\
Z_{0} & \left.=\frac{120 \pi}{\sqrt{\varepsilon \text { reff }}\left[\frac{w}{h}+1.393+0.667 \ln \left(\frac{w}{h}+1.444\right)\right.}\right]
\end{aligned}
$$

Figure 3 shows the final antenna geometry on FR4 substrate along with dimensions and feed point. Pink area shows the radiating layout and green area shows the substrate region.

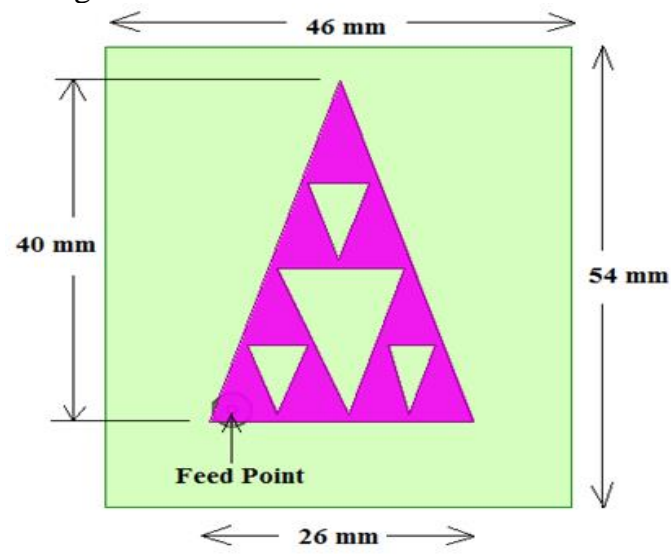

Fig 3. Geometry description of the proposed fractal antenna.

Side view of complete antenna layout is shown in Figure 4. It clearly shows an entire assembly together with fractal radiator, ground, substrate and coaxial connector. Compactness of this planar antenna will be calculable from this figure. Dimensional details of the designed antenna is given in Table 1, units used in the dimensional calculations are in millimeters

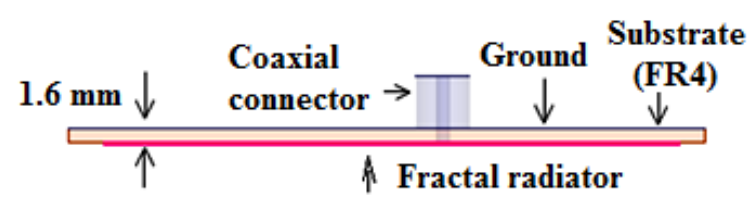

Fig 4. Complete structural details including connector placement and both sides.

Table 1 Layout details of the designed antenna.

\begin{tabular}{|c|c|}
\hline Antenna Design Parameters & Values (mm) \\
\hline Height \& Width of FR4 & $54 \times 46$ \\
\hline Thickness & 1.6 \\
\hline H \& W of ground & $54 \times 46$ \\
\hline Width of Sierpinski Patch & 26 \\
\hline Height of Sierpinski Patch & 40 \\
\hline
\end{tabular}

\section{OUTCOME AND CONVERSE}

The proposed antenna was first designed on varied substrates like Arlon (6.15), Bakelite (4.8), Polyester (3.2) however the most effective results are obtained on FR4 epoxy (4.4) substrate only. The Simulated $S_{11}$ graph is shown in Figure 5. Voltage standing wave ratio for all resonating frequencies lies in admissible range $\leq 2$ [4].

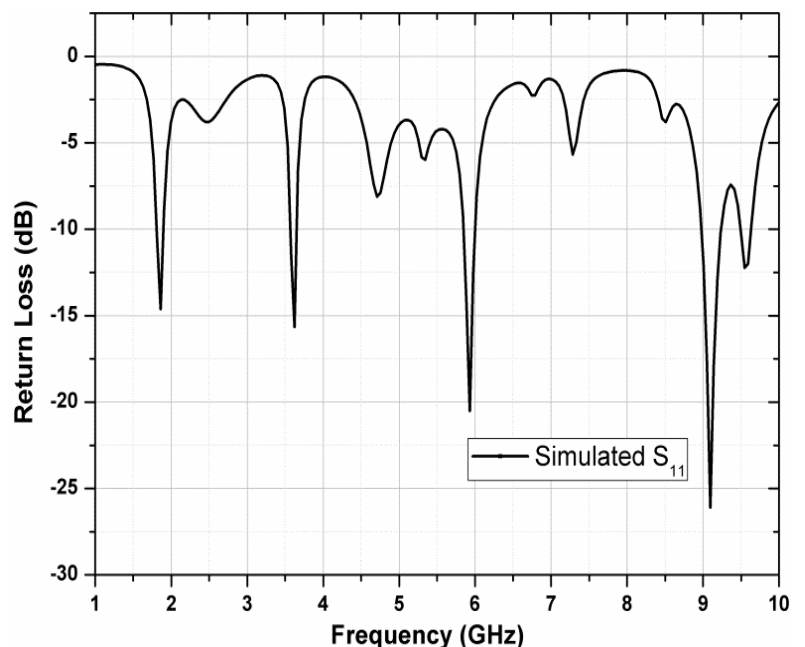

Fig 5. Simulated return loss $\left(S_{11}\right)$ of proposed antenna for FR4 substrate.

Suggested antenna resonates at five different frequencies $1.859 \mathrm{GHz}, 3.623 \mathrm{GHz}, 5.929 \mathrm{GHz}, 9.095 \mathrm{GHz}$ and 9.547 GHz. Figure 6 (a to e) shows the simulated 2Dimensional radiation diagrams for all the resonating frequencies. All these patterns depicts directional behavior of the antenna in the far-field region which is found to be almost omnidirectional in $H$-plane and bidirectional in $E$ plane. Fluctuation within the pattern observed in the radiation pattern as we tend to elevate frequency limit. 
The reason behind this is found to be reflection of the radiated signal at the edges and divergence in the flow of current from standing wave patterns [15].

3-Dimensional radiation diagram along with gain legend is revealed in fig. 7. A low profile gain of $3 \mathrm{~dB}$ (max) is also observed. Table 2 shows the detailed allocation of all the resonating frequencies ' $\mathrm{Fr}$ ' along with their respective VSWR values and associated bandwidth for each frequency band.

Table 2. Distribution of resonating frequencies.

\begin{tabular}{|c|c|c|c|c|}
\hline Sr. No & $\begin{array}{c}\text { Fr } \\
\text { (GHz) }\end{array}$ & S $_{11}(\mathbf{d b})_{\max }$ & $\begin{array}{c}\text { B.W } \\
\text { (MHz) }\end{array}$ & VSWR \\
\hline 1 & 1.859 & -14.6 & 86.5 & 1.45 \\
\hline 2 & 3.623 & -15.64 & 84.1 & 1.39 \\
\hline 3 & 5.929 & -20.5 & 154 & 1.2 \\
\hline 4 & 9.095 & -26.09 & 253 & 1.1 \\
\hline 5 & 9.547 & -12.24 & 14 & 1.64 \\
\hline
\end{tabular}

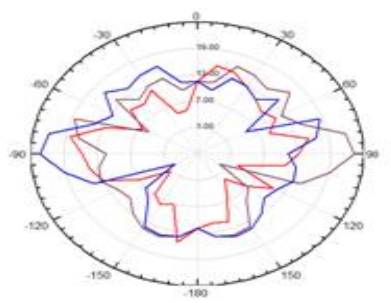

(a)

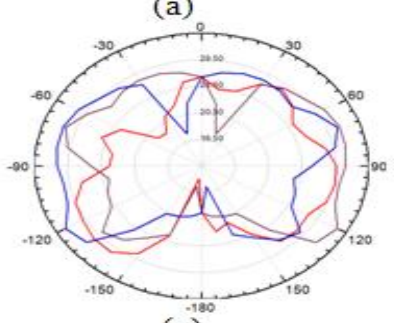

(c)

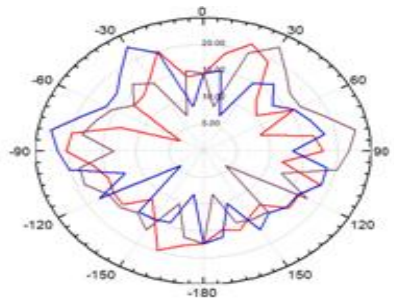

(b)

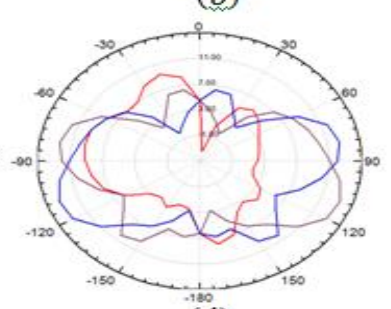

(d)

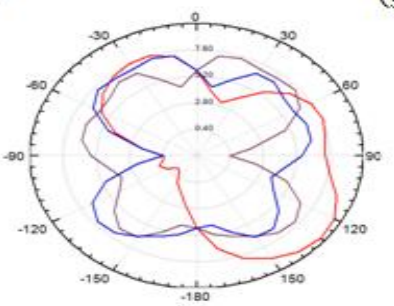

(e)

Fig 6. 2-Dimensional radiation diagrams for all 5 resonating frequencies.

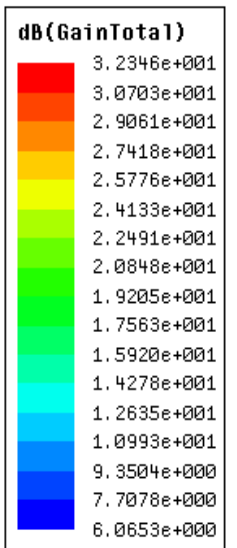

Fig 7. Three dimensional radiation distribution in free space along with gain legend.

\section{CONCLUSION}

A redesigned Sierpinski fractal antenna based on Sierpinski gasket is intended and imitated on the FR4 epoxy substrate and the findings are scrutinized. Multiple resonance situations are determined by fractal layouts observed and are brought into play to procure additional resonance situations. The complete antenna layout has compact geometry of $54 \mathrm{~mm} \times 46 \mathrm{~mm} \times 1.6 \mathrm{~mm}$ and exhibits penta-band of operation by resonating at five different frequencies $1.859 \mathrm{GHz}, 3.623 \mathrm{GHz}, 5.929 \mathrm{GHz}$, $9.095 \mathrm{GHz}$, and $9.547 \mathrm{GHz}$ along with acceptable values of reflection coefficient, gain, voltage standing wave ratio, and bandwidth. It finds its utility in compact wireless devices due to its lightweight, compact size and planar geometry.

\section{REFERENCES}

1. J. S. J. Anguera, C. Puente, and C. Borja, "Fractal-Shaped Antennas: Review," Wiley Encyclopedia of RF and Microwave Engineering, vol. 2, pp. 1620-1635, 2005.

2. J. Pourahmadazar, C. Ghobadi, J. Nourinia, and H. Shirzad, "Multiband ring fractal monopole antenna for mobile devices," IEEE Antennas and Wireless Propagation Letters, vol. 9, no. 1, pp. 863-866, 2010.

3. C. T. P. Song, "Fractal antenna research at University of Birmingham," vol. 2000, pp. 724-727, 2005

4. C. E. Balanis, Antenna Theory: Analysis and Design, 3rd Edition Constantine A. Balanis. John Wiley \& Sons, 2005

5. K. C. Hwang, "A Modified Sierpinski Fractal Antenna for Multiband Application," IEEE Antennas and Wireless Propagation Letters, vol. 6, pp. 357-360, 2007.

6. P. Ciais, R. Staraj, G. Kossiavas, and C. Luxey, "Design of an internal quad-band antenna for mobile phones," IEEE Microwave and Wireless Components Letters, vol. 14, no. 4, pp. 148-150, 2004.

7. B. B. Mandelbrot, "The Fractal Geometry of Nature," American Journal of Physics, vol. 51, no. 3, p. 286, 1983

8. J. Anguera, E. Martínez, C. Puente, C. Borja, and J. Soler, "Broad-band dual-frequency microstrip patch antenna with modified Sierpinsk fractal geometry," IEEE Transactions on Antennas and Propagation, vol. 52, no. 1, pp. 66-73, 2004.

9. Y. M. Madany and H. Elkamchouchi, "Analysis of high gain multiband rounded corners dashed rectangular spiral microstrip patch antenna," IEEE Antennas and Propagation Society, AP-S International Symposium (Digest), vol. 1 A, no. 1, pp. 313-316, 2005.

10. W. Peng, W. Anguo, and D. Jiawei, "Design of the UWB antenna using fractal concept," ISAPE 2008 - The 8th International Symposium on Antennas, Propagation and EM Theory Proceedings, pp. 189-192, 2008.

11. M. F. Abd Kadir, A. S. Ja'afar, and M. Z. A. Abd Aziz, "Sierpinski carpet fractal antenna," 2007 Asia-Pacific Conference on Applied Electromagnetics Proceedings, APACE2007, no. 2, pp. 1-4, 2007.

12. T. Zeybek and K. ElMahgoub, "A Dual Band Modified Sierpinski Antenna for WiFi Applications," in 2018 IEEE International Symposium on Antennas and Propagation \& USNC/URSI National Radio Science Meeting, 2018, pp. 733-734.

13. P. N. Rao and N. V. S. N. Sarma, "The Effect of Indentation Angle of Koch Fractal Boundary on the Performance of Microstrip Antenna," International Journal of Antennas and Propagation, vol. 2008, pp. 1-5 2008.

14. C. P. Baliarda, C. B. Borau, M. N. Rodero, and J. R. Robert, "An iterative model for fractal antennas: application to the Sierpinski gasket antenna," IEEE Transactions on Antennas and Propagation, vol. 48, no. 5, pp. 713-719, May 2000.

15. M. R. Jena, M. B.B, and D. Mishra, "Bandwidth and Gain Enhancement of Multiband Fractal Antenna Based on the Sierpinski Carpet Geometry," ICTACT Journal on Communication Technology, vol. 04, no. 01, pp. 669-674, 2016. 


\section{Coaxial Probe Fed Modified Sierpinski Fractal Antenna for Wireless Applications}

\section{AUTHORS PROFILE}

Yadwinder Kumar born in Punjab, India on March 1979 and presently working as Sr. Assistant Professor in YCOE, Talwandi Sabo (Regional campus of Punjabi University, Patiala) Distt. Bathinda, Punjab, in the department of Electronics \& Communication Engineering. Mr. Kumar is a lifetime member of IMS (Indian Microelectronics Society) and actively working in the field of Hybrid Fractal Antennas and Microelectronics. He has done his B.Tech in Electronics \& Communication Engineering from G.N.D.E.C, Ludhiana, in 2001, M.Tech in Microelectronics from UCIM, Panjab University, Chandigarh in 2005 and Ph.D from SLIET longowal in 2016. 